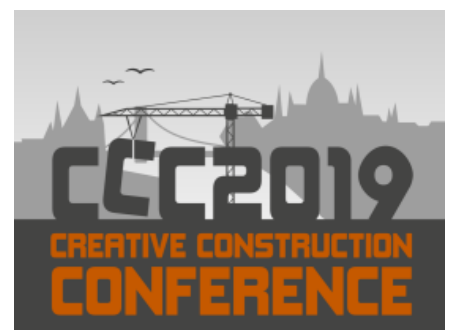

Available online at 2019.creative-construction-conference.com/proceedings/

CCC 2019

Proceedings of the Creative Construction Conference (2019) 051

Edited by: Miroslaw J. Skibniewski \& Miklos Hajdu

https://doi.org/10.3311/CCC2019-051

Creative Construction Conference 2019, CCC 2019, 29 June - 2 July 2019, Budapest, Hungary

\title{
Improved Unbalanced Bid Detection Model
}

\author{
Gul Polat ${ }^{\mathrm{a}, *}$, Harun Turkoglu ${ }^{\mathrm{b}}$, Atilla Damcic $^{\mathrm{c}}$, Firat Dogu Akin ${ }^{\mathrm{b}}$ \\ ${ }^{a}$ Professor, Istanbul Technical Universiy, Istanbul 34469, Turkey \\ ${ }^{b}$ Research Assistant, Istanbul Technical Universiy, Istanbul 34469, Turkey \\ ${ }^{c}$ Associate Professor, Istanbul Technical Universiy, Istanbul 34469, Turkey
}

\begin{abstract}
Detection of unbalanced bids is crucial for owners because selecting an unbalanced bidder as the contractor may bring about cost overruns. There are two main types of unbalanced bids, namely, mathematically and materially unbalanced bid. This study mainly focuses on the second type, where a contractor tends to increase the unit prices of items whose quantity was somehow underrated by the owner's team. This study proposes a modification to a model that was developed to assist owners in detecting unbalanced bids. The major difference between the proposed model and the previous one lies in the grading system of detecting the unbalanced bids. In the proposed model, eight different grading systems are used in detection of unbalanced bids, whereas the previous model consisted of five grading systems. The final score of each bidder is calculated by assigning weights to these grading systems. Bidders are evaluated not only according to the offered bid prices, but also according to the calculated final scores. The applicability of the proposed approach is presented along with an illustrative example. It was observed that the proposed model detected the unbalanced bid, which attains the lowest final score. The proposed model represents a marked improvement on existing practice and provides owners with a new perspective in detecting unbalanced bids. Armed with such a tool, it may be easier for owners to protect themselves from the risk of unbalanced bids.
\end{abstract}

(C) 2019 The Authors. Published by Budapest University of Technology and Economics \& Diamond Congress Ltd.

Peer-review under responsibility of the scientific committee of the Creative Construction Conference 2019.

Keywords: Unbalanced bid; detection model; grading system; owner; case study.

\section{Introduction}

Cost overrun is one of the main problems frequently encountered in construction projects [1]. From the owner's perspective, one of the effective ways to prevent cost overrun is to detect unbalanced bids during the bid evaluation phase. Manipulation of bid items' prices without changing the total bid price can be called as "unbalanced bidding" [2]. There are two main methods of unbalancing a bid: mathematically (front-end loading) and materially (quantity error exploitation) [3,4]. Front-loading is a method that increases the unit prices of the activities scheduled to start in the early phases of the project while reducing the unit prices of the activities scheduled to start in the later phases. Thus, contractors can achieve better cash flows and higher profits while reducing their financial risks in the project [3]. End-loading is a method that is applied by increasing unit prices of activities in the later phases of the project. End-loading is a less-used method compared to front-loading. End-loading method is usually used for taking advantage of the escalation in countries with high inflation rates [4]. Quantity error exploitation is a method applied by increasing the unit prices of the activities in which actual quantities are expected to exceed the estimated ones, while decreasing the unit prices of the activities that are overestimated [5].

*Corresponding author: Gul Polat email: polatgu@itu.edu.tr 
Polat et al. / Proceedings of the Creative Construction Conference (2019) 051

https://doi.org/10.3311/CCC2019-051

Unbalanced bidding in the construction industry have been discussed by several researchers thoroughly [3]. Although detecting unbalanced bids created by quantity error exploitation is more difficult for owners [6], and there are few studies that help owners to detect and prevent unbalanced bids, most of the studies in the literature are conducted from the perspective of contractors [2,4-9]. Therefore, this study focused on the detection of unbalanced bids created by quantity error exploitation in unit price contracts. For this purpose, after reviewing the existing models in the literature, an advanced unbalanced bid detection model was proposed by improving the model developed by Polat et al., (2018) [10]. The proposed model uses eight different grading systems in detection of unbalanced bids, whereas the previous model consisted of five grading systems. Owners may assign different weights to these grading systems according to the characteristics of their projects. After assigning weights to the grading systems, the final scores of each bidder can be calculated. All bidders can be evaluated not only according to their bid prices but also according to the calculated final scores. An illustrative example is presented to check the applicability of the proposed model in construction projects. The findings of this study indicated that the proposed model provided a marked improvement compared to the previous model. It also provides owners with a new perspective in detecting unbalanced bids during the bid evaluation phase.

\section{The Improved Unbalanced Bid Detection Model}

The objectives of this study include: (1) defining new grading systems to extend the existing approach in detection of unbalanced bids, (2) validating the new grading systems through an illustrative example. In order to achieve these objectives, the model developed by Polat et al., (2018) was modified by adding new grading systems. For the new grading systems, major and minor bid items have been defined. If a bid item's total price is equal to or greater than five percent of the construction cost estimated (ECC) by the owner, this bid item called as a "major bid item". On the other hand, if a bid item's total price is less than five percent of the ECC, this bid item called as a "minor bid item". Polat et al., (2018) described the existing grading systems for detection of unbalanced bids. The modified approach proposed in this study adopts the same grading systems used by Polat et al., (2018), but the following grading systems are added for detection of unbalanced bids.

Sixth grading system: The main idea behind this grading system is to compare the unit price of each major activity $i_{m j}$ $\left(i_{m j}=1,2, \ldots, n\right)\left(\right.$ bupi $\left._{m j}\right)$ offered by each bidder with the ones estimated by the owner (oupi $\left.i_{m j}\right)$. The ratio of each major activity's total price $\left(\right.$ or $\left._{i m j}\right)$ estimated by the owner in the ECC is calculated using Equation 2. In this grading system, the comparison ratio $\left(r_{6}\right)$ is calculated using Equation 1. Bidders receive a grade for each major activity $\left(g_{6 i m j}\right)$ based on the intervals given in Table 1. The total score received from the sixth grading system $\left(B T S_{6}\right)$ is found by using Equation 3, where $g_{\max }$ is the maximum value of the sixth grading system $\left(g_{\max }=42\right)$.

$$
\begin{aligned}
& r_{6}=\frac{\operatorname{bup}_{i_{m j}}}{\operatorname{oup}_{i_{m j}}} \\
& o r_{i_{m j}}=\frac{\operatorname{oup}_{i_{m j}} \times q_{i_{m j}}}{E C C} \\
& \text { BTS }_{6}=\frac{\sum_{i_{m j}=1}^{n}\left(\text { or }_{i_{m j}} \times g_{6 i_{m j}}\right)}{\sum_{i_{m j}=1}^{n}\left(o r_{i_{m j}}\right) \times g_{\max }} \times 100
\end{aligned}
$$

Seventh grading system: The main idea behind this grading system is to compare the unit price of each major activity

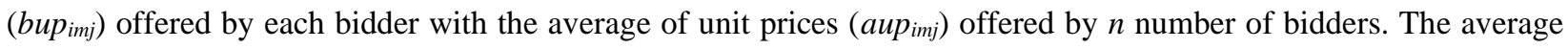
unit price of each major activity and the comparison ratio $\left(r_{7}\right)$ are calculated using Equation 4 and 5, respectively. Bidders receive a grade $\left(g_{7 \mathrm{imj}}\right)$ according to the comparison ratio obtained for each major activity (see Table 1$)$. Then, 
Polat et al. / Proceedings of the Creative Construction Conference (2019) 051

https://doi.org/10.3311/CCC2019-051

the total score received from the seventh grading system $\left(B T S_{7}\right)$ is found using Equation 6, where $g_{\max }$ is the maximum value of the seventh grading system $\left(g_{\max }=42\right)$.

$$
\begin{aligned}
& \operatorname{aup}_{i_{m j}}=\frac{\operatorname{bup}_{1}+\operatorname{bup}_{2+} \ldots+\text { bup }_{n}}{n} \\
& r_{7}=\frac{\operatorname{bup}_{i_{m j}}}{\operatorname{aup}_{i_{m j}}} \\
& \text { BTS }_{7}=\frac{\sum_{i_{m j}=1}^{n}\left(\text { or }_{i_{m j}} \times g_{7 i_{m j}}\right)}{\sum_{i_{m j}=1}^{n}\left(\text { or }_{i_{m j}}\right) \times g_{\max }} \times 100
\end{aligned}
$$

Eighth grading system: The main idea behind this grading system is to compare the ratio of sum of the major activities' total prices $\left(b r_{i m j}\right)$ to that of minor activities $\left(b r_{i m n}\right)$ with the ones $\left(o r_{i m j}\right.$, or $\left.r_{i m n}\right)$ estimated by the owner (see Equations 7-8). The comparison ratio $\left(r_{8}\right)$ is calculated by Equation 9. Bidders receive a grade $(g 8)$ according to the comparison ratio presented in Table 1 . The total score for the eighth grading system $\left(B T S_{8}\right)$ is calculated using Equation 10, where $\left(g_{\max }=42\right)$.

$$
\begin{aligned}
& b r_{i_{m j}}=\sum_{i_{m j}=1}^{n}\left(\operatorname{bup}_{i_{m j}} \times q_{i_{m j}}\right), \text { br }_{i_{m n}}=\sum_{i_{m n}=1}^{n}\left(\text { bup }_{i_{m n}} \times q_{i_{m n}}\right) \\
& o r_{i_{m j}}=\sum_{i_{m j}=1}^{n}\left(\operatorname{oup}_{i_{m j}} \times q_{i_{m j}}\right), o r_{i_{m n}}=\sum_{i_{m n}=1}^{n}\left(\operatorname{oup}_{i_{m n}} \times q_{i_{m n}}\right)
\end{aligned}
$$

where $q_{i m j}$ is the quantity of major activity $i_{m j}$ and $q_{i m n}$ is the quantity of minor activity $i_{m n}$.

$$
\begin{aligned}
& r_{8}=\frac{\left(b r_{i_{m j}} \div b r_{i_{m n}}\right)}{\left(o r_{i_{m j}} \div o r_{i_{m n}}\right)} \\
& B T S_{8}=\frac{g_{8}}{g_{\max }} \times 100
\end{aligned}
$$

For all grading systems, a comparison rate is calculated. Bidders receive grades depending on these ratios. The grading table (Table 1) allows the owner to evaluate the bidders objectively. In grading systems 6, 7, and 8, a comparison rate that is higher than 1.050 corresponds to the lowest grade $\left(g_{\min }=1\right)$, whereas a comparison rate lower than 0.950 corresponds to the highest grade $\left(g_{\max }=42\right)$. Finally, each bidder's final score can be calculated by assigning weights to each grading system according to a preferred criterion (Equation 11). The evaluation of the bidders will be based on these final scores.

$$
F S=\sum_{j=1}^{8}\left(w_{j} \times B T S_{j}\right)
$$

where $w_{j}$ is the weight for the $j^{\text {th }}$ grading system and $B T S_{j}$ is the total score for the $j^{\text {th }}$ grading system. 
Polat et al. / Proceedings of the Creative Construction Conference (2019) 051 https://doi.org/10.3311/CCC2019-051

Table 1. Grade values for grading system 6,7 and 8.

\begin{tabular}{llllllll}
\hline Comparison Ratio & Grade & Comparison Ratio & Grade & Comparison Ratio & Grade & Comparison Ratio & Grade \\
\hline $\mathrm{r} \leq 0.9$ & 42 & $0.950<\mathrm{r} \leq 0.955$ & 31 & $1.005<\mathrm{r} \leq 1.010$ & 20 & $1.060<\mathrm{r} \leq 1.065$ & 9 \\
$0.900<\mathrm{r} \leq 0.905$ & 41 & $0.955<\mathrm{r} \leq 0.960$ & 30 & $1.010<\mathrm{r} \leq 1.015$ & 19 & $1.065<\mathrm{r} \leq 1.070$ & 8 \\
$0.905<\mathrm{r} \leq 0.910$ & 40 & $0.960<\mathrm{r} \leq 0.965$ & 29 & $1.015<\mathrm{r} \leq 1.020$ & 18 & $1.070<\mathrm{r} \leq 1.075$ & 7 \\
$0.910<\mathrm{r} \leq 0.915$ & 39 & $0.965<\mathrm{r} \leq 0.970$ & 28 & $1.020<\mathrm{r} \leq 1.025$ & 17 & $1.075<\mathrm{r} \leq 1.080$ & 6 \\
$0.915<\mathrm{r} \leq 0.920$ & 38 & $0.970<\mathrm{r} \leq 0.975$ & 27 & $1.025<\mathrm{r} \leq 1.030$ & 16 & $1.080<\mathrm{r} \leq 1.085$ & 5 \\
$0.920<\mathrm{r} \leq 0.925$ & 37 & $0.975<\mathrm{r} \leq 0.980$ & 26 & $1.030<\mathrm{r} \leq 1.035$ & 15 & $1.085<\mathrm{r} \leq 1.090$ & 4 \\
$0.925<\mathrm{r} \leq 0.930$ & 36 & $0.980<\mathrm{r} \leq 0.985$ & 25 & $1.035<\mathrm{r} \leq 1.040$ & 14 & $1.090<\mathrm{r} \leq 1.095$ & 3 \\
$0.930<\mathrm{r} \leq 0.935$ & 35 & $0.985<\mathrm{r} \leq 0.990$ & 24 & $1.040<\mathrm{r} \leq 1.045$ & 13 & $1.095<\mathrm{r} \leq 1.100$ & 2 \\
$0.935<\mathrm{r} \leq 0.940$ & 34 & $0.990<\mathrm{r} \leq 0.995$ & 23 & $1.045<\mathrm{r} \leq 1.050$ & 12 & $1.100<\mathrm{r}$ & 1 \\
$0.940<\mathrm{r} \leq 0.945$ & 33 & $0.995<\mathrm{r} \leq 1.000$ & 22 & $1.050<\mathrm{r} \leq 1.055$ & 11 & & \\
$0.945<\mathrm{r} \leq 0.950$ & 32 & $1.000<\mathrm{r} \leq 1.005$ & 21 & $1.055<\mathrm{r} \leq 1.060$ & 10 & & \\
\hline
\end{tabular}

\section{Illustrative Example}

An illustrative example used by Polat et al., (2018) is presented to validate the applicability of the proposed model in construction projects. The presented example compromises 72 activities, and 5 out of 72 activities are identified as "major activities" while others are identified as "minor activities". The unit price of each activity estimated by the owner are taken from "The Construction and Installation Unit Prices Book" published by the Turkish Ministry of Environment and Urban Planning. The units, quantities, unit prices of these 72 activities estimated by the owner $(\mathrm{O})$ and offered by 8 bidders (B) are presented in Table 2 .

Table 2. Input data for illustrative example.

\begin{tabular}{|c|c|c|c|c|c|c|c|c|c|c|c|}
\hline \multirow{2}{*}{$\begin{array}{l}\text { Act. } \\
\text { ID }\end{array}$} & \multirow{2}{*}{ Unit } & \multirow{2}{*}{ Quantity } & \multicolumn{9}{|c|}{ Unit Prices (TL - Turkish Liras) } \\
\hline & & & $\mathbf{O}$ & $\mathbf{B}_{1}$ & $\mathbf{B}_{2}$ & $\mathbf{B}_{3}$ & $\mathbf{B}_{4}$ & $\mathbf{B}_{5}$ & $\mathbf{B}_{6}$ & $\mathbf{B}_{7}$ & $\mathbf{B}_{8}$ \\
\hline $\mathrm{A}_{1}$ & $\mathrm{~m}^{3}$ & 700 & 14.38 & 14.75 & 13.07 & 15.50 & 15.56 & 14.92 & 13.64 & 14.88 & 14.66 \\
\hline $\mathrm{A}_{2}$ & $\mathrm{~m}^{3}$ & 365 & 38.83 & 41.91 & 41.25 & 42.38 & 35.58 & 41.11 & 38.14 & 40.41 & 36.32 \\
\hline $\mathrm{A}_{3}$ & $\mathrm{~m}^{3}$ & 850 & 2.84 & 3.08 & 2.59 & 2.96 & 2.63 & 2.98 & 2.84 & 2.86 & 2.68 \\
\hline $\mathrm{A}_{4}$ & $\mathrm{~m}^{3}$ & 736 & 4.83 & 5.29 & 4.57 & 5.27 & 5.15 & 4.36 & 4.46 & 4.62 & 4.72 \\
\hline $\mathrm{A}_{5}$ & $\mathrm{~m}$ & 198 & 67.70 & 65.94 & 63.94 & 74.14 & 65.43 & 69.76 & 68.75 & 69.39 & 62.33 \\
\hline $\mathrm{A}_{6}$ & $\mathrm{~m}^{3}$ & 59 & 31.88 & 29.82 & 28.96 & 29.66 & 29.41 & 33.12 & 29.21 & 28.89 & 29.34 \\
\hline $\mathrm{A}_{7}$ & $\mathrm{~m}^{3}$ & 150 & 14.19 & 13.60 & 13.21 & 14.34 & 13.67 & 13.78 & 15.17 & 13.64 & 15.11 \\
\hline $\mathrm{A}_{8}$ & $\mathrm{~m}^{3}$ & 90 & 29.19 & 26.97 & 28.68 & 29.95 & 31.42 & 30.40 & 31.12 & 29.52 & 30.38 \\
\hline $\mathrm{A}_{9}$ & $\mathrm{~m}^{3}$ & 2000 & 178.63 & 170.44 & 183.53 & 173.77 & 166.04 & 189.94 & 187.99 & 175.84 & 169.66 \\
\hline $\mathrm{A}_{10}$ & $\mathrm{~m}$ & 1200 & 335.43 & 330.84 & 362.48 & 336.29 & 333.25 & 322.75 & 318.94 & 329.43 & 353.08 \\
\hline $\mathrm{A}_{11}$ & $\mathrm{~m}$ & 650 & 68.40 & 65.53 & 74.79 & 69.38 & 63.26 & 64.79 & 69.13 & 72.10 & 73.25 \\
\hline $\mathrm{A}_{12}$ & $\mathrm{~m}^{3}$ & 350 & 52.20 & 51.26 & 47.00 & 54.89 & 56.72 & 47.53 & 54.14 & 52.51 & 56.04 \\
\hline $\mathrm{A}_{13}$ & $\mathrm{~m}^{3}$ & 100 & 86.29 & 84.53 & 92.11 & 85.72 & 91.16 & 88.14 & 85.52 & 88.90 & 82.79 \\
\hline $\mathrm{A}_{14}$ & $\mathrm{~m}^{3}$ & 360 & 121.63 & 112.82 & 133.19 & 131.99 & 130.73 & 122.52 & 128.27 & 111.87 & 124.88 \\
\hline $\mathrm{A}_{15}$ & $\mathrm{~m}$ & 36 & 29.19 & 29.64 & 28.97 & 30.16 & 27.98 & 28.77 & 31.89 & 29.44 & 27.92 \\
\hline $\mathrm{A}_{16}$ & $\mathrm{~m}$ & 40 & 33.40 & 35.59 & 34.05 & 32.36 & 36.04 & 35.12 & 36.57 & 32.56 & 36.23 \\
\hline $\mathrm{A}_{17}$ & $\mathrm{~m}^{2}$ & 1000 & 22.18 & 20.98 & 23.41 & 20.95 & 23.78 & 22.35 & 23.19 & 23.00 & 20.95 \\
\hline $\mathrm{A}_{18}$ & $\mathrm{~m}^{2}$ & 750 & 23.24 & 23.34 & 21.41 & 23.86 & 21.85 & 21.32 & 24.63 & 21.88 & 22.87 \\
\hline $\mathrm{A}_{19}$ & $\mathrm{~m}^{2}$ & 635 & 31.39 & 32.96 & 31.66 & 29.76 & 30.56 & 32.36 & 28.90 & 29.47 & 33.33 \\
\hline $\mathrm{A}_{20}$ & $\mathrm{~m}^{2}$ & 400 & 35.64 & 36.75 & 37.55 & 36.58 & 39.03 & 35.38 & 37.56 & 35.40 & 36.21 \\
\hline $\mathrm{A}_{21}$ & $\mathrm{~m}^{2}$ & 348 & 38.05 & 39.78 & 38.77 & 39.28 & 35.44 & 35.79 & 39.19 & 39.63 & 35.08 \\
\hline $\mathrm{A}_{22}$ & $\mathrm{~m}^{2}$ & 250 & 50.16 & 50.34 & 49.73 & 52.14 & 45.85 & 52.27 & 49.12 & 50.30 & 49.46 \\
\hline $\mathrm{A}_{23}$ & $\mathrm{~m}^{2}$ & 100 & 26.56 & 26.26 & 27.36 & 26.88 & 25.07 & 24.98 & 27.50 & 24.59 & 27.82 \\
\hline
\end{tabular}

Table 2 (cont'd). Input data for illustrative example.

\begin{tabular}{|c|c|c|c|c|c|c|c|c|c|c|c|}
\hline \multirow{2}{*}{$\begin{array}{l}\text { Act. } \\
\text { ID }\end{array}$} & \multirow{2}{*}{ Unit } & \multirow{2}{*}{ Quantity } & \multicolumn{9}{|c|}{ Unit Prices (TL - Turkish Liras) } \\
\hline & & & $\mathbf{O}$ & $\mathbf{B}_{1}$ & $\mathbf{B}_{2}$ & $\mathbf{B}_{3}$ & $\mathbf{B}_{4}$ & $\mathbf{B}_{5}$ & $\mathbf{B}_{6}$ & $\mathbf{B}_{7}$ & $\mathbf{B}_{8}$ \\
\hline $\mathrm{A}_{24}$ & $\mathrm{~m}^{2}$ & 150 & 35.63 & 36.26 & 34.12 & 37.72 & 34.84 & 35.66 & 35.98 & 32.82 & 35.03 \\
\hline $\mathrm{A}_{25}$ & $\mathrm{~m}^{2}$ & 75 & 23.61 & 24.87 & 21.25 & 21.81 & 24.29 & 23.15 & 23.41 & 24.72 & 24.89 \\
\hline
\end{tabular}


Polat et al. / Proceedings of the Creative Construction Conference (2019) 051 https://doi.org/10.3311/CCC2019-051

\begin{tabular}{|c|c|c|c|c|c|c|c|c|c|c|c|}
\hline $\mathrm{A}_{26}$ & $\mathrm{~m}^{2}$ & 98 & 28.59 & 25.89 & 26.65 & 28.53 & 28.52 & 31.41 & 28.69 & 28.21 & 30.46 \\
\hline $\mathrm{A}_{27}$ & $\mathrm{~m}^{2}$ & 50 & 27.29 & 27.41 & 25.95 & 29.84 & 26.82 & 28.77 & 25.32 & 25.65 & 24.73 \\
\hline $\mathrm{A}_{28}$ & $\mathrm{~m}^{2}$ & 43 & 29.98 & 30.20 & 29.84 & 27.50 & 30.75 & 28.48 & 32.57 & 28.26 & 30.09 \\
\hline $\mathrm{A}_{29}$ & $\mathrm{~m}^{2}$ & 66 & 44.61 & 45.92 & 47.46 & 44.67 & 40.65 & 48.92 & 42.76 & 43.53 & 41.53 \\
\hline $\mathrm{A}_{30}$ & $\mathrm{~m}^{2}$ & 40 & 58.94 & 54.01 & 56.03 & 53.11 & 59.19 & 59.65 & 54.99 & 59.98 & 60.40 \\
\hline$A_{31}$ & $\mathrm{~m}^{2}$ & 40 & 39.54 & 43.20 & 42.80 & 39.07 & 37.86 & 38.69 & 42.66 & 41.16 & 39.66 \\
\hline $\mathrm{A}_{32}$ & $\mathrm{~m}^{2}$ & 100 & 40.24 & 42.55 & 39.59 & 41.02 & 42.52 & 43.04 & 41.36 & 41.56 & 36.69 \\
\hline $\mathrm{A}_{33}$ & $\mathrm{~m}^{2}$ & 450 & 1.94 & 1.75 & 2.08 & 2.01 & 1.94 & 2.00 & 1.99 & 2.01 & 1.75 \\
\hline $\mathrm{A}_{34}$ & $\mathrm{~m}^{2}$ & 350 & 2.35 & 2.17 & 2.48 & 2.39 & 2.27 & 2.55 & 2.20 & 2.58 & 2.19 \\
\hline $\mathrm{A}_{35}$ & $\mathrm{~m}^{2}$ & 40 & 16.91 & 15.70 & 15.29 & 15.72 & 16.19 & 15.85 & 15.66 & 16.05 & 17.10 \\
\hline $\mathrm{A}_{36}$ & $\mathrm{~m}^{2}$ & 60 & 20.71 & 18.91 & 20.89 & 20.97 & 20.51 & 21.01 & 22.31 & 20.95 & 20.96 \\
\hline $\mathrm{A}_{37}$ & $\mathrm{~m}^{2}$ & 50 & 14.68 & 15.50 & 14.91 & 13.82 & 13.55 & 13.29 & 14.37 & 14.57 & 13.84 \\
\hline $\mathrm{A}_{38}$ & $\mathrm{~m}^{2}$ & 1000 & 27.71 & 26.86 & 27.56 & 26.32 & 29.55 & 26.55 & 26.78 & 27.64 & 28.43 \\
\hline $\mathrm{A}_{39}$ & $\mathrm{~m}^{2}$ & 450 & 43.24 & 39.04 & 46.78 & 45.19 & 46.00 & 43.59 & 45.01 & 45.12 & 47.13 \\
\hline $\mathrm{A}_{40}$ & $\mathrm{~m}^{2}$ & 900 & 32.39 & 34.31 & 29.90 & 34.25 & 33.58 & 32.77 & 29.28 & 31.71 & 29.34 \\
\hline $\mathrm{A}_{41}$ & $\mathrm{~m}^{2}$ & 650 & 33.90 & 33.90 & 30.71 & 36.87 & 36.48 & 30.83 & 35.46 & 31.94 & 31.03 \\
\hline $\mathrm{A}_{42}$ & $\mathrm{~m}^{2}$ & 100 & 6.29 & 6.84 & 5.71 & 6.33 & 5.92 & 5.68 & 5.97 & 6.86 & 6.02 \\
\hline $\mathrm{A}_{43}$ & $\mathrm{~m}^{2}$ & 1000 & 1.29 & 1.29 & 1.31 & 1.23 & 1.40 & 1.36 & 1.22 & 1.19 & 1.18 \\
\hline $\mathrm{A}_{44}$ & $\mathrm{~m}^{2}$ & 150 & 7.33 & 7.01 & 6.67 & 6.64 & 7.45 & 7.91 & 7.12 & 7.29 & 7.64 \\
\hline $\mathrm{A}_{45}$ & $\mathrm{~m}^{2}$ & 2000 & 11.78 & 11.58 & 12.45 & 11.39 & 11.86 & 12.38 & 11.66 & 12.90 & 12.94 \\
\hline $\mathrm{A}_{46}$ & $\mathrm{~m}^{2}$ & 1600 & 30.04 & 29.74 & 30.87 & 31.84 & 27.61 & 30.66 & 31.46 & 29.77 & 30.38 \\
\hline $\mathrm{A}_{47}$ & $\mathrm{~m}^{2}$ & 2000 & 29.56 & 30.55 & 30.33 & 30.01 & 32.43 & 28.17 & 30.23 & 29.49 & 27.07 \\
\hline $\mathrm{A}_{48}$ & $\mathrm{~m}^{3}$ & 600 & 4.59 & 4.65 & 4.72 & 4.72 & 4.68 & 4.50 & 4.27 & 4.87 & 4.15 \\
\hline $\mathrm{A}_{49}$ & $\mathrm{~m}^{3}$ & 450 & 5.84 & 5.36 & 6.42 & 5.29 & 5.88 & 6.11 & 5.41 & 6.06 & 5.64 \\
\hline $\mathrm{A}_{50}$ & $\mathrm{~m}^{2}$ & 750 & 4.83 & 5.29 & 4.61 & 4.97 & 5.18 & 4.77 & 4.79 & 4.86 & 5.21 \\
\hline $\mathrm{A}_{51}$ & $\mathrm{~m}^{2}$ & 1600 & 115.81 & 107.85 & 117.90 & 125.97 & 116.49 & 107.78 & 122.21 & 120.78 & 120.28 \\
\hline $\mathrm{A}_{52}$ & $\mathrm{~m}^{2}$ & 650 & 136.51 & 142.73 & 139.82 & 133.89 & 126.59 & 128.11 & 149.12 & 141.47 & 126.39 \\
\hline $\mathrm{A}_{53}$ & $\mathrm{~m}^{2}$ & 650 & 88.36 & 89.02 & 81.44 & 91.52 & 92.22 & 93.89 & 89.25 & 79.90 & 81.71 \\
\hline $\mathrm{A}_{54}$ & $\mathrm{~m}^{2}$ & 250 & 123.24 & 133.28 & 134.02 & 112.88 & 133.04 & 134.99 & 119.83 & 131.82 & 114.27 \\
\hline $\mathrm{A}_{55}$ & $\mathrm{~m}^{2}$ & 690 & 50.34 & 48.72 & 47.31 & 53.42 & 49.00 & 46.54 & 53.07 & 51.53 & 45.89 \\
\hline $\mathrm{A}_{56}$ & $\mathrm{~m}^{2}$ & 600 & 170.88 & 157.50 & 161.31 & 160.47 & 179.91 & 166.13 & 182.67 & 164.95 & 182.67 \\
\hline $\mathrm{A}_{57}$ & $\mathrm{~m}^{2}$ & 350 & 319.38 & 338.86 & 338.76 & 344.12 & 339.48 & 325.54 & 350.95 & 306.97 & 302.08 \\
\hline $\mathrm{A}_{58}$ & $\mathrm{~m}^{2}$ & 400 & 250.09 & 261.30 & 253.70 & 264.69 & 249.89 & 244.55 & 265.10 & 264.46 & 226.00 \\
\hline $\mathrm{A}_{59}$ & ton & 1300 & 2096.56 & 2127.33 & 2152.31 & 1941.54 & 2089.57 & 2077.30 & 2026.49 & 1988.62 & 2045.57 \\
\hline $\mathrm{A}_{60}$ & ton & 1650 & 2017.94 & 2140.37 & 2143.31 & 1877.71 & 1975.24 & 1998.95 & 1887.98 & 2160.53 & 1974.92 \\
\hline $\mathrm{A}_{61}$ & ton & 350 & 1972.66 & 1871.37 & 1796.76 & 2120.92 & 2169.36 & 1789.12 & 2155.85 & 1837.37 & 1987.05 \\
\hline $\mathrm{A}_{62}$ & ton & 1000 & 1939.23 & 1985.11 & 1832.36 & 1762.37 & 1999.50 & 2115.62 & 2044.00 & 1860.08 & 1918.36 \\
\hline $\mathrm{A}_{63}$ & ton & 1150 & 1914.79 & 1914.13 & 1780.31 & 2038.14 & 1781.33 & 1987.31 & 1875.30 & 1810.11 & 1885.75 \\
\hline $\mathrm{A}_{64}$ & ton & 200 & 3386.01 & 3642.50 & 3635.81 & 3425.98 & 3236.22 & 3658.23 & 3346.48 & 3591.23 & 3238.26 \\
\hline $\mathrm{A}_{6}$ & $\mathrm{~kg}$ & 4000 & 8.64 & 9.39 & 8.07 & 8.58 & 7.87 & 8.65 & 9.13 & 8.38 & 7.97 \\
\hline $\mathrm{A}_{66}$ & $\mathrm{~m}^{2}$ & 2000 & 9.59 & 9.16 & 10.29 & 10.06 & 9.74 & 10.16 & 9.55 & 8.82 & 10.51 \\
\hline$A_{67}$ & $\mathrm{~m}^{2}$ & 600 & 13.00 & 12.96 & 13.32 & 14.30 & 14.00 & 13.68 & 11.84 & 12.36 & 14.07 \\
\hline $\mathrm{A}_{68}$ & $\mathrm{~m}^{2}$ & 150 & 5.23 & 5.62 & 5.34 & 5.53 & 5.32 & 5.06 & 4.91 & 5.17 & 5.45 \\
\hline $\mathrm{A}_{69}$ & $\mathrm{~m}^{2}$ & 2000 & 15.65 & 14.26 & 16.26 & 14.72 & 16.25 & 15.96 & 14.87 & 15.75 & 16.47 \\
\hline $\mathrm{A}_{70}$ & $\mathrm{~m}^{2}$ & 2000 & 18.56 & 19.20 & 16.81 & 18.13 & 19.78 & 18.95 & 17.37 & 17.73 & 18.78 \\
\hline $\mathrm{A}_{71}$ & $\mathrm{~m}^{2}$ & 700 & 28.60 & 27.78 & 27.36 & 26.28 & 31.23 & 30.24 & 27.97 & 27.13 & 28.49 \\
\hline $\mathrm{A}_{72}$ & $\mathrm{~m}^{2}$ & 2000 & 20.88 & 21.98 & 22.75 & 22.95 & 19.38 & 19.91 & 21.36 & 19.67 & 22.51 \\
\hline
\end{tabular}

The construction cost $(E C C)$ estimated by the owner is 13,766,619.41 TL, and the bid prices offered by 8 bidders are $14,043,276.86 \mathrm{TL}\left(B P_{l}\right), 13,826,569.14 \mathrm{TL}\left(B P_{2}\right), 13,389,997.59$ TL $\left(B P_{3}\right), 13,624,850.19 \mathrm{TL}\left(B P_{4}\right), 13,947,114.50$ $\mathrm{TL}\left(B P_{5}\right), 13,622,893.85 \mathrm{TL}\left(B P_{6}\right), 13,641,083.17 \mathrm{TL}\left(B P_{7}\right)$, and 13,538,572.61 TL $\left(B P_{8}\right)$, respectively. The weights assigned to grading systems are different from the weights used in Polat et al.'s (2018) study due to the addition of three new grading systems to the proposed model. In this study, weights were assigned as $15 \%$ for first grading system, $10 \%$ for the second one, $5 \%$ for the third one, $10 \%$ for the fourth one, $30 \%$ for the fifth one, $5 \%$ for the sixth one, $5 \%$ for the seventh one, and 5\% for the eighth one. The owner can assign weights to each grading system according to the 
Polat et al. / Proceedings of the Creative Construction Conference (2019) 051 https://doi.org/10.3311/CCC2019-051

project characteristics. The ranking of the bidders according to the final scores is presented in Table 3. Based on the results presented in Table 3, Bidder $2\left(B_{2}\right)$ has the highest final score, whereas Bidder $1\left(B_{1}\right)$ has the lowest final score. Although $B_{3}$ offered the lowest bid price, it is ranked sixth according to the final scores. Moreover, $B_{1}$ has the lowest final score while offering the highest bid price. Finally, $B_{1}$ submits the highest bid price and the most unbalanced bid, whereas $B_{2}$ submits the most balanced bid despite not offering the lowest bid price. In other words, $B_{2}$ is the most appropriate bidder for the owner. The findings of this study reveal that the improved model provided a different ranking of bidders than the one obtained by Polat et al.'s (2018) model.

Table 3. Rankings of the bidders.

\begin{tabular}{lllllllll}
\hline \multicolumn{1}{c}{ Bidders } & $\mathbf{B}_{\mathbf{1}}$ & $\mathbf{B}_{\mathbf{2}}$ & $\mathbf{B}_{\mathbf{3}}$ & $\mathbf{B}_{\mathbf{4}}$ & $\mathbf{B}_{\mathbf{5}}$ & $\mathbf{B}_{\mathbf{6}}$ & $\mathbf{B}_{\mathbf{7}}$ & $\mathbf{B}_{\mathbf{8}}$ \\
\hline Proposed Models & 6 & 2 & 8 & 3 & 7 & 4 & 5 & 1 \\
Previous unbalanced bid detection model * & 6 & 1 & 6 & 4 & 7 & 3 & 5 & 2 \\
\hline Improved unbalanced bid detection model & 8 & & & & & & &
\end{tabular}

\section{Conclusion}

The main aim of this study is to propose an improved version of the unbalanced bid detection model developed by Polat et al. (2018). The proposed model uses eight different grading systems. Owners may assign weights to each grading system according to the characteristics of their projects. After assigning weights to each grading system, the final scores of bidders can be calculated. All bidders can be evaluated not only according to the offered bid prices but also according to the calculated final scores. An illustrative example is presented to validate the applicability of the proposed model. It was observed that the improved model detected the unbalanced bid but provided a different ranking of bidders than the one obtained by Polat et al.'s (2018) model. However, this study is limited as it only focuses on unbalanced bids created by quantity error exploitation method in unit price contracts. In future studies, the model can be developed so that it can deal with the detection of unbalanced bids created by different methods.

\section{References}

[1] Enshassi, A., Al-Najjar, J., Kumaraswamy, M., Delays and cost overruns in the construction projects in the Gaza Strip, Journal of Financial Management of Property and Construction, (2009), 14(2), 126-151. https://doi.org/10.1108/13664380910977592

[2] Hyari, K. H., Tarawneh, Z. S., Katkhuda, H. N., Detection Model for Unbalanced Pricing in Construction Projects: A Risk-Based Approach, Journal of Construction Engineering and Management, (2016), 142(12), 04016078. https://doi.org/10.1061/(ASCE)CO.1943-7862.0001203

[3] Christodoulou, S. E., A bid-unbalancing method for lowering a contractor's financial risk. Construction Management and Economics, (2008), 26(12), 1291-1302. https://doi.org/10.1080/01446190802596238

[4] Arditi, D., Chotibhongs, R., Detection and Prevention of Unbalanced Bids, Construction Management and Economics, (2009), $27(8), 721$ - 732. https://doi.org/10.1080/01446190903117785

[5] Hyari, K. H., Handling unbalanced bidding in construction projects: Prevention rather than detection. Journal of Construction Engineering and Management, (2015), 142(2), 04015060. https://doi.org/10.1061/(ASCE)CO.1943-7862.0001045

[6] Nikpour, B., Senouci, A., Eldin, N., Detection Tool for Unbalanced Bids, Open Journal of Civil Engineering, (2017), 7(03), 409. https://doi.org/10.4236/ojce.2017.73028

[7] Cattell, D. W., Bowen, P. A., Kaka, A. P., Review of Unbalanced Bidding Models in Construction, Journal of Construction Engineering and Management, (2007), 133(8), 562-573. https://doi.org/10.1061/(ASCE)0733-9364(2007)133:8(562)

[8] An, X., Li, H., Zuo, J., Ojuri, O., Wang, Z., Ding, J., Identification and Prevention of Unbalanced Bids Using the Unascertained Model. Journal of Construction Engineering and Management, (2018), 144(11), 05018013. https://doi.org/10.1061/(ASCE)CO.1943-7862.0001563

[9] Hyari, K. H., Owner's countermeasures to skewed bidding in construction projects: Review of current practices and proposal for new countermeasures, Journal of Management in Engineering, (2017), 33(3), 04016053. https://doi.org/10.1061/(ASCE)ME.1943-5479.0000502

[10] Polat, G., Turkoglu, H., Damci, A. (2018). Detection of unbalanced bids: A case study, Creative Construction Conference 2018, Ljubljana, Slovenia, (2018), pp. 432-439. https://doi.org/10.1061/(ASCE)0733-9364(2007)133:8(562) 